\title{
Chromosomal rearrangements and copy number abnormalities of TP63 correlate with p63 protein expression in lung adenocarcinoma
}

Marie-Christine Aubry ${ }^{1}$, Anja Roden ${ }^{1}$, Stephen J Murphy ${ }^{2}$, George Vasmatzis ${ }^{2}$, Sarah H Johnson ${ }^{2}$, Faye R Harris ${ }^{2}$, Geoffrey Halling ${ }^{2}$, Ryan A Knudson ${ }^{1}$, Rhett P Ketterling ${ }^{1}$ and Andrew L Feldman ${ }^{1}$

${ }^{1}$ Departments of Laboratory Medicine and Pathology, Mayo Clinic, Rochester, MN, USA and ${ }^{2}$ Department of Molecular Medicine, Mayo Clinic, Rochester, MN, USA

The TP63 gene encodes a member of the p53 family of transcription factors. Although TP53 is a well-known tumor suppressor gene, the role of p63 in tumorigenesis is controversial. Our group recently identified novel chromosomal rearrangements involving TP63 in approximately $6 \%$ of peripheral T-cell lymphomas, which correlated with a p63 $+/$ p40 - immunohistochemical profile. As a subset of lung adenocarcinomas are p63 $+I$ p40 - , we undertook the current study to examine the presence of TP63 rearrangements and correlate with p63/ p40 expression. Next-generation sequencing was used to identify genomic rearrangements of TP63 in 37 adenocarcinomas. Confirmatory fluorescence in-situ hybridization (FISH) using a break-apart probe to the TP63 gene region and immunohistochemistry for p63 and p40 were performed on adenocarcinomas with TP63 rearrangements identified by mate-pair sequencing. Immunohistochemistry for p63 and p40 was performed on 45 additional adenocarcinomas, and FISH was performed on all adenocarcinomas with p63 positivity. TP63 rearrangement was identified in two adenocarcinoma specimens from a single patient. The rearrangement resulted in a complex rearrangement of $3 q$ that fused B3GALNT1 at the $3^{\prime}$ intron to TP63. FISH confirmed the rearrangement in both tumors. Immunohistochemistry staining for p63 was diffuse ( $>80 \%$ cells + ) and p40 was negative. Of the 44 additional adenocarcinomas, $13(30 \%)$ showed p63 expression; p40 was negative in all cases. No case showed rearrangement of TP63 by a break-apart FISH. However, extra copies of the intact TP63 locus were seen in the p63-positive areas of all 12 cases, with copy numbers ranging from three to seven. We have identified a novel chromosomal rearrangement involving TP63 in a p63+/p40 - lung adenocarcinoma. Break-apart FISH testing can be used to diagnose this finding. Immunohistochemistry for p63 was not specific for this rearrangement, as nearly $33 \%$ of adenocarcinomas expressed p63. Additional copies of the intact TP63 locus were also a common finding and correlated with immunohistochemistry positivity for p63.

Modern Pathology (2015) 28, 359-366; doi:10.1038/modpathol.2014.118; published online 5 September 2014

The TP63 gene, located on chromosome 3q28, encodes a member of the p53 family of transcription factors. ${ }^{1}$ The p63 protein has a critical role in the maintenance of progenitor cells in epithelial development and morphogenesis. Indeed, Trp63 knockout mice show severe developmental defects, such as

Correspondence: Dr M-C Aubry, MD, Departments of Laboratory Medicine and Pathology, Mayo Clinic, 200 First Street SW, Rochester, MN 55905, USA.

E-mail: aubry.mariechristine@mayo.edu

Received 7 April 2014; revised 14 July 2014; accepted 30 July 2014; published online 5 September 2014 lack of limbs and tissues such as teeth and mammary glands. ${ }^{2}$ Furthermore, these mice do not develop complete stratified epithelium. Human TP63 variants most commonly encompass single-nucleotide point mutations and have been reported in various genetic syndromes, including Acro-dermal-ungual-lacrimaltooth syndrome, ectrodactyly-ectodermal dysplasia and cleft lip/palate syndrome, Hay-Wells syndrome, and limb-mammary syndrome. ${ }^{2}$ Interestingly, none of these syndromes are tumor prone.

Although TP53 is a well-known tumor suppressor gene and its role in tumorigenesis has been extensively studied, the role of p63 in tumorigenesis 
has been more controversial. Based on in vitro and in vivo mouse studies, full-length p63 containing the $\mathrm{N}$-terminal transactivation domain (TAp63) shares the most homology with p53 and acts as a tumor suppressor gene, inducing growth suppression and death. ${ }^{2-4}$ In contrast, p63 isoforms with N-terminal truncations $(\Delta \mathrm{Np} 63)$ may have either tumor suppressor or oncogenic function, depending on the genes that are transactivated. ${ }^{5,6}$ Several mouse models with Trp63 mutations are tumor prone. The tumors are of various types, including carcinomas and sarcomas, and usually are metastatic, indicating aggressive behavior. ${ }^{6}$

TP53 is commonly mutated in human cancers; in contrast, mutations in TP63 have rarely been reported, including in lung cancer $(<4 \%$ of nonsmall-cell carcinomas studied). ${ }^{3,5,7,8}$ Most are missense mutations reportedly with a tumor suppressor activity. ${ }^{3,8}$ These mutations have been described in both squamous cell carcinoma and adenocarcinoma.

However, TP63 amplification and immunohistochemical nuclear expression is common in many cancers. ${ }^{5,9}$ In lung cancer, amplification and expression is most commonly observed in squamous cell carcinoma, and p63 by immunohistochemistry has been routinely used to distinguish squamous cell carcinoma from adenocarcinoma. ${ }^{10-12}$ However, amplification has been reported in a small subset (11\%) of adenocarcinoma and expression consistently observed in up to $32 \%$ of adenocarcinoma. ${ }^{13-15}$ Usually p63 expression in adenocarcinoma is focal but in about $5 \%$ of tumors, expression is more diffuse. ${ }^{16,17}$ Most antibodies raised against p63, including the 4A4 clone commonly used for immunohistochemistry, do not distinguish between the transactivated and $\Delta \mathrm{N}$ isoforms of p63; the proposed explanation for the detection of p63 expression in adenocarcinoma. In contrast, an antibody against p40, which targets the $\Delta \mathrm{N}$ isoforms of p63, has been shown to be highly specific for squamous cell carcinoma and rarely reported in adenocarcinoma. ${ }^{15,17}$

Using next-generation sequencing analysis of mate-pair DNA libraries, our group recently identified novel chromosomal rearrangements involving TP63 in approximately $6 \%$ in peripheral T-cell lymphomas and demonstrated that these were associated with a poorer overall survival than lymphomas without these rearrangements. ${ }^{18}$ These rearrangements also were found in occasional B-cell lymphomas, but were not identified by RNA sequencing in a small series of carcinomas of the lung, breast, or head and neck. ${ }^{18,19}$ By immunohistochemistry, TP63 rearrangements were associated with a p63 $+/ \mathrm{p} 40-$ profile in lymphomas. ${ }^{18}$ As a subset of lung adenocarcinomas has a p63+/p40 - immunoprofile, we examined the presence of TP63 rearrangements and associated p63/p40 expression of lung adenocarcinomas.

\section{Materials and methods}

\section{Case Selection}

Thirty seven lung adenocarcinomas were sequenced using the mate-pair break-point sequencing protocol (see below). For this study, data were mined for TP63 genomic rearrangements. Fluorescence in-situ hybridization (FISH) and immunohistochemistry were used to further evaluate potential TP63 rearrangements. A validation study was performed on a separate cohort of 45 resected adenocarcinoma including immunohistochemistry for p63 and p40. All cases positive for p63 and negative for p40 and two cases negative for both were selected for further FISH studies. The diagnosis of adenocarcinoma was confirmed using the WHO classification ${ }^{20}$ and classified according to the new proposed classification of adenocarcinoma. ${ }^{21}$ The Mayo Clinic Institutional Review Board approved the study.

\section{DNA Isolation and Mate-pair Sequencing}

Frozen lung adenocarcinoma sections were cut into 10-micron size and pure cell populations were isolated using the Arcturus PixCell II microscope and CapSure Macro Laser Capture Microdissection caps (Arcturus; LCM 0211). Associated histologically non-neoplastic tissue was also collected by laser capture microdissection from adjacent benign tissue blocks associated with each case. Whole-genome amplification (WGA) was performed directly on laser captured microdissected cells using a single-step procedure. ${ }^{22}$ Briefly, laser captured microdissected cells were incubated for $10 \mathrm{~min}$ in $0.5 \mathrm{X}$ Repli-g D2 buffer (6.5 $\mu \mathrm{l}$ ) (Qiagen, CA, USA) and then in Repli-g stop solution $(3.5 \mu \mathrm{l})$. Repli-g mini kit Master Mix $(40 \mu \mathrm{l})$ was then added and incubated at $30^{\circ} \mathrm{C}$ for $16 \mathrm{~h}$. Four individual $50 \mu \mathrm{l}$ WGA reactions were pooled for each sample. DNA was quantified by Quant-iT PicoGreen analysis (Invitrogen, P7581). Mate-Pair libraries were assembled from WGA DNA according to a previously published protocol ${ }^{22}$ using the Illumina mate-pair kit. Briefly, WGA DNA (10 $\mu \mathrm{g})$ was fragmented to $\sim 3 \mathrm{~kb}$ and DNA intramolecularcircles assembled by ligation following biotin-end labeling. Additional fragmentation to $350-650 \mathrm{bp}$ was followed by immobilization of biotinylated terminal fragments on M-280 streptavidin beads (Dynal) and assembly of adapter flanked Illuminaindexed paired-end libraries using Illumina adapters (Illumina, CA, USA). Two multiplexed libraries were loaded per lane of an Illumina flow cell and sequenced to $101 \times 2$ paired-end reads on an Illumina HiSeq. Base calling was performed using Illumina Pipeline v1.5.

\section{Sequencing Alignment and Bioinformatic Analysis}

Bioinformatic protocols to rapidly and efficiently process Next-Generation Sequencing Mate-Pair data 
using a 32-bit binary indexing of the hg19 reference genome, to which consecutive 32-bit binary sequences from associated Mate Pair reads are aligned, have been previously published from our laboratory. ${ }^{18,23}$ The algorithm maps both Mate-Pair reads successively to the whole genome, selecting reads $<15 \mathrm{~kb}$ apart allowing up to 10 mismatches, with the lowest cumulative mismatch count sent to the output. Discordant Mate-Pairs mapping $>15 \mathrm{~kb}$ apart or to different chromosomes were selected for further analysis and associated fragments were clustered together. False positives were eliminated using algorithmic filters based on homology scores calculated during mapping. Replication was calculated for each chromosome and replicate read-pairs were removed. Bridged coverage was calculated as the sum of the fragment lengths (distance between read 1 and read 2) of correctly mapping Mate-Pair and Pair-End reads, divided by the mappable chromosome size. Base coverage was calculated from the total number of mappable reads, multiplied by the read length and divided by the mappable chromosome size.

\section{PCR Validation}

Primers were designed flanking the predicted TP63 break point $\left(5^{\prime}\right.$-GAATGAAGGA ATGAAATACC CTCAGATGAC-3'; chromosome 3, 160,819,243$160,819,272$ and 5 -CTATGAAAAA GCTGGTGCTC ATCAGACG-3'; chromosome 3, 189,430,262$189,430,289)$. Validation PCR $(25 \mu \mathrm{l}$ reaction volumes, $50 \mathrm{ng}$ Template, 35 cycles) was performed using the EasyA high fidelity polymerase (Stratagene, La Jolla, CA, USA, \#600404) on tumor DNA from the adenocarcinomas (AD1 and AD2), nonneoplastic lung from the same patient and a human Genomic DNA control (G304A; Promega, Madison, WI, USA), as well as germline blood DNA (B). PCR products were visualized on $1 \%$ agarose gel and excised/extracted DNA was sequenced by standard Sanger sequencing protocols using both forward and reverse primers.

\section{Immunohistochemistry for p63 and p40}

Four micron sections cut from formalin-fixed, paraffin-embedded blocks were placed on 'charged' slides; slides were then dried and melted in a $62{ }^{\circ} \mathrm{C}$ oven for $20 \mathrm{~min}$. Slides were stained with mouse monoclonal p63 antibody (clone BC4A4, Biocare, Concord, CA, USA) at a 1/250 titer, or with rabbit polyclonal p40 antibody (Biocare) at a 1/100 titer. Slides were placed on a Ventana BenchMark XT (Ventana Medical Systems, Oro Valley, AZ, USA) for staining. The staining protocol included online deparaffinization, Heat Induced Epitope Retrieval with Ventana Cell Conditioning 1 for 32 mins, primary antibody incubation for $16 \mathrm{mins}$ (p63) or 32 mins (p40) at $37^{\circ} \mathrm{C}$. Antigen-antibody reactions were visualized using the Ventana OptiViewTM Universal DAB Detection Kit. Counterstaining was performed online using Ventana Hematoxylin II for 8 mins, followed by bluing reagent for 4 mins. All slides were removed from the stainer, dehydrated, and coverslipped for microscopic examination. Appropriate positive and negative controls were used.

Results were reported as percent of tumor cells with nuclear staining, and only cases with $>5 \%$ positive cells were considered positive. If present, cytoplasmic staining for p63 was noted.

\section{FISH}

Interphase FISH was performed on formalin-fixed paraffin-embedded sections of lung adenocarcinoma and controls using a break-apart probe to the TP63 gene region as previously described. ${ }^{18}$ Briefly, sections were deparaffinized and dehydrated, pretreated with Tris/EDTA and $\mathrm{NaCl}$ protease, and hybridized with probes flanking the TP63 gene and fluorescently labeled with Spectrum Orange $\left(5^{\prime}\right)$ or Spectrum Green $\left(3^{\prime}\right)$. Slides were analyzed for abnormal separation of red and green fluorescent signals using standard fluorescence microscopy techniques. In cases with a heterogeneous staining pattern for p63 by immunohistochemistry, p63positive and p63-negative areas of the tumor were circled and scored separately in the FISH analysis.

\section{Results}

Of the 37 sequenced adenocarcinomas, a TP63 rearrangement was identified in two (5\%) synchronous lung tumors from one patient (Figure 1). This patient underwent resection of two adenocarcinomas with a 5 months interval, the first from the right middle lobe and the second from the left lower lobe. Histologically, both tumors were mucinous adenocarcinoma and determined to be intrapulmonary metastasis through shared genomic break points. The TP63 fusion resulted from a complex rearrangement on the q-arm of chromosome 3 that fused the B3GALNT1 gene at locus 3q26.1a to TP63 at locus $3 q 28 b$ (Figure 1a). The rearrangement predicted an inversion of the gene sequence in the third intron of B3GALNT1 transcript variant 1, which fused with a region in the first intron of TP63 transcript variant 1 , positioning the promoter of B3GALNT1 in line with the TP63 coding unit from exon 2 onwards (Figure 1b). PCR validation of the rearrangement using primers flanking the predicted fusion junction generated unique bands in both adenocarcinomas, which was absent in non-neoplastic lung tissue from the same patient and a mixed germline population control sample (Figure 1c). Additional validation in germline DNA extracted from blood from that patient also confirmed the event as a novel somatic rearrangement (Figure 1d). Sanger sequencing on 


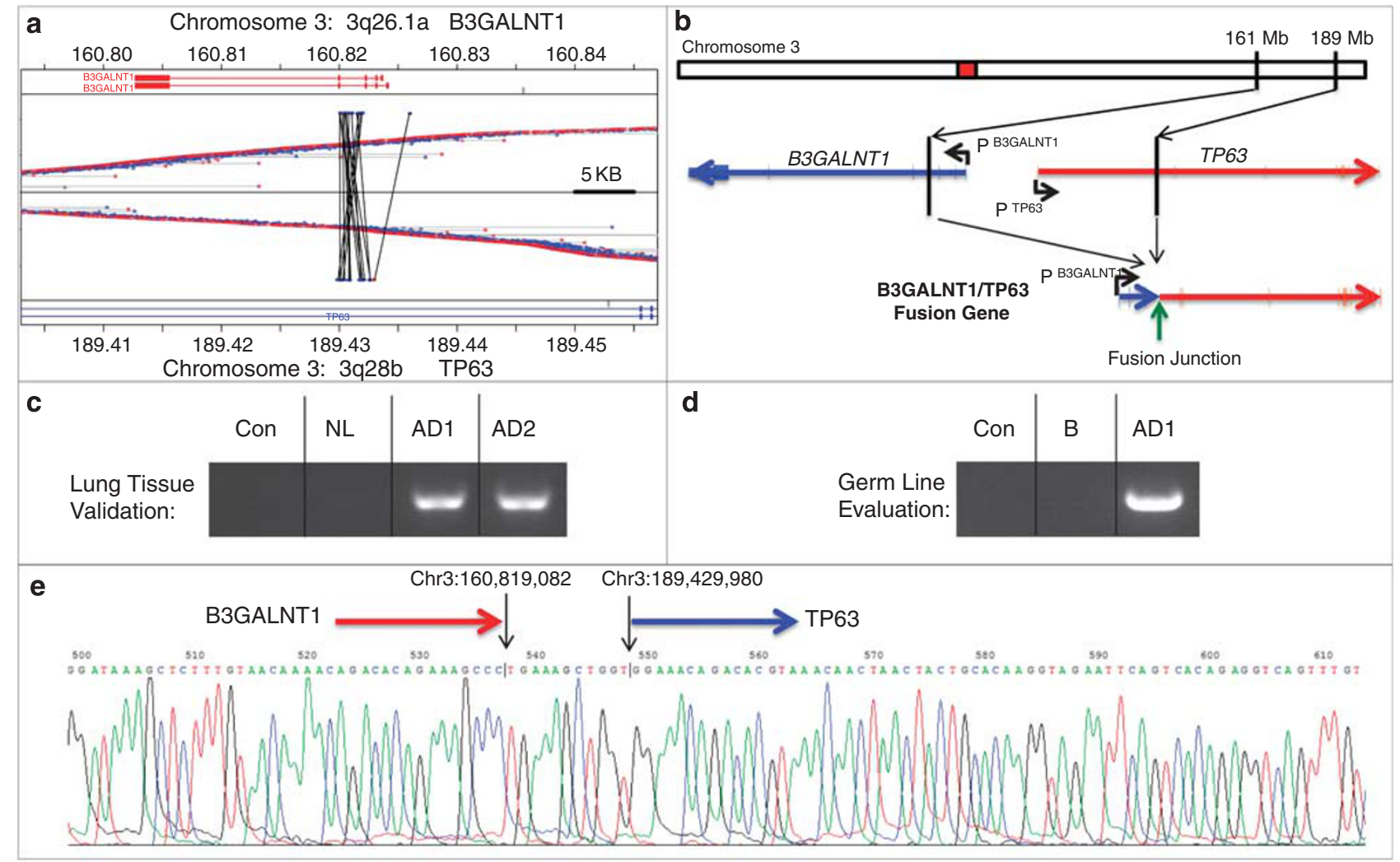

Figure 1 (a) Junction plot of Mate-Pair data showing inversion chr3:(q26-q28) involving B3GALNT1 and TP63. Regions 3q26.1 and $3 q 28 \mathrm{~b}$ are presented on the upper and lower parts of the plot, respectively. Mate-pair reads are mapped to the genome positions as red or blue dots, according to their mapping polarities to the positive or negative DNA strands, respectively. Normal concordantly mapping mate-pair reads are presented as red/blue linked arrays moving vertically from each region. Discordantly mapping mate-pair reads are presented as dots with linked lines spanning across the upper and lower represented genomic areas. (b) Schematic representation of the predicted B3GALNT1/TP63 fusion. (c) PCR validation on adenocarcinomas (AD1 and AD2), non-neoplastic lung (NL), and mixed population germline control (Con). (d) Additional PCR validation on blood-derived germline DNA (B). (e) Sanger sequencing profile showing fusion junction.

DNA extracted from the PCR validation bands confirmed the fusion with precise positions at chromosome 3, 160,819,605 and 189,429,980, with an intermediate 11 nucleotides between these fusion positions of unknown origin (Figure 1e). Immunohistochemistry staining for p63 was diffuse ( $>80 \%$ tumor cells + ) and p40 was negative in both adenocarcinomas (Figure 2a-c). Break-apart FISH confirmed rearrangement of TP63 in both samples (Figure 2d).

Of the 45 additional adenocarcinomas, 13 of 44 (one technical failure, $30 \%$ ) showed p63 expression by immunohistochemistry in $20-60 \%$ of cells; p40 was negative in all cases (Table 1). None of the cases showed cytoplasmic staining. FISH was performed in 12 of these 13 cases (one had insufficient tissue). No case showed rearrangement of TP63 by breakapart FISH. However, extra copies of the intact TP63 locus were seen in the p63-positive areas of all 12 cases, with copy numbers ranging from 3 to 7 (Figure 3). In four cases, the areas of immunohistochemical positivity for p63 were sufficiently discrete that FISH could be scored separately in the p63-positive and p63-negative areas. Although all p63-positive areas demonstrated extra copies of TP63, only two copies of TP63 were present in the p63-negative areas in three of four cases. FISH was also performed in two adenocarcinomas negative for both p63 and p40, and only two copies of TP63 were found.

The TP63 rearrangement was identified in a multifocal mucinous adenocarcinoma. Further studies of two additional mucinous adenocarcinomas failed to show p63 expression. P63 expression was less common in lepidic predominant adenocarcinoma than other adenocarcinoma subtypes (Table 1).

\section{Discussion}

To our knowledge, this is the first report of chromosomal rearrangements of TP63 in lung cancer, specifically in lung adenocarcinoma. Using the same strategy as in peripheral T-cell lymphoma, ${ }^{18}$ we first identified a $3 q 26-28$ chromosomal 

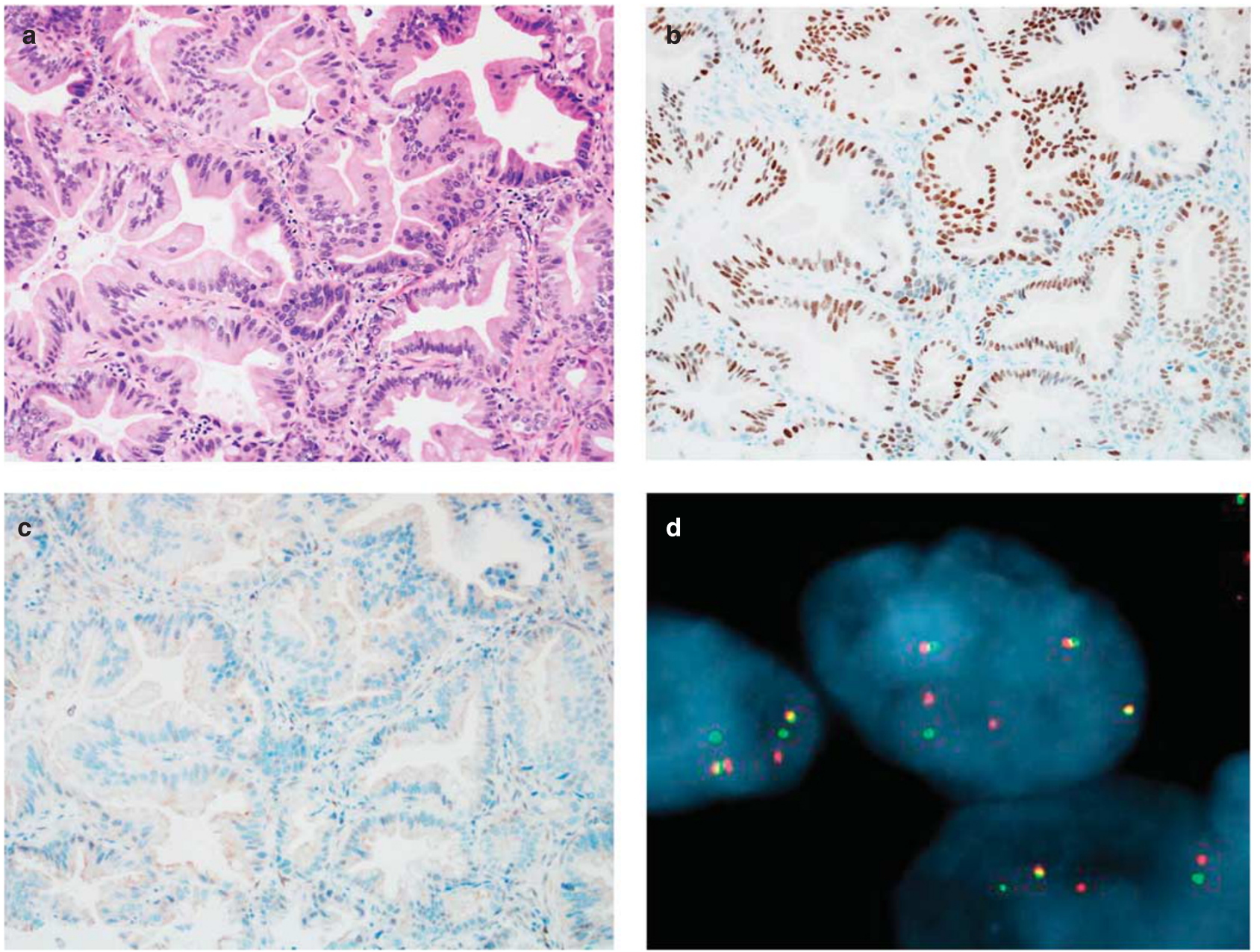

Figure 2 (a) Intermediate power magnification of one of the mucinous adenocarcinomas with TP63 rearrangement $(\mathrm{H} \& \mathrm{E}, \times 200)$. (b) The adenocarcinoma is diffusely positive for p63 (p63, $\times 200$ ), (c) and negative for p40 (p40, $\times 200)$. (d) The break-apart FISH for p63 show an abnormal separation of the orange and green fluorescent signals indicative of the chromosomal rearrangement.

rearrangement in sequenced lung adenocarcinoma, which validated using PCR and Sanger sequencing. We further confirmed the rearrangements using a break-apart FISH probe, and also performed immunohistochemistry for p63 and p40. Thus far, TP63 genomic rearrangements had been described only in lymphomas. ${ }^{18,19}$ In diffuse large B-cell lymphomas, TP63 is fused to TBL1XR1. This fusion is seen in 5\% of diffuse large B-cell lymphomas, exclusively of germinal center type. ${ }^{19}$ The authors hypothesized that the fusion was functionally similar to the $\Delta$ Np63, potentially providing a proliferative advantage and resistance to genotoxic stress induced by chemotherapy. Vasmatzis et al ${ }^{18}$ reported TP63 rearrangements in 11 (5.8\%) peripheral T-cell lymphomas, seven of which had TBL1XR1 as a partner, and in two $(1.2 \%)$ diffuse large B-cell lymphomas. The authors hypothesized that TP63 rearrangements may represent an alternate route of deregulating the p53 pathway in peripheral T-cell lymphoma. Interestingly, the authors did not detect any TP63 fusions using the Snow-Shoes-FTD algorithm in transcriptome sequencing data in the studied lung cancers. However, the histologic subtypes of these lung cancers were unknown.

In our patient's tumors, the partner to TP63 is B3GALNT1. This gene is a member of the $\beta-1,3-$ galactosyltransferase gene family which encodes for membrane-bound glycoproteins with various enzymatic functions. According to the catalog of somatic mutations in cancer, mutations in B3GALNT1 are uncommon $(0.4 \%)$ and mostly missense. In the Cancer Genome Atlas database, four mutations (three noncoding and one missense) were identified in adenocarcinoma and one (noncoding) in squamous cell carcinoma. No fusion transcript involving this gene has been reported. The role of TP63 rearrangement in lung carcinogenesis still remains to be elucidated. The findings of TP63 amplification and p63 expression in lung cancer suggest a role for TP63 in cancer development. Indeed, TP63 amplification is present in high-grade dysplasia/squamous cell carcinoma in situ but not in low-grade dysplasia. ${ }^{14}$ Similarly, p63 expression progressively 
increases from pre-neoplastic to pre-invasive and invasive squamous cell carcinoma. ${ }^{13}$

The clinical significance of a chromosomal rearrangement of TP63 in lung adenocarcinoma will need to be studied in a large cohort of patients as the frequency of this finding appears low, seen in only one $(1 \%)$ patient from our cohort of 81 patients, although this is a frequency similar to other clinically relevant genomic events in lung cancer. In lymphomas, TP63 rearrangements are associated with adverse outcomes. In diffuse large B-cell lymphoma, three of five TP63 rearranged cases were refractory to R-CHOP chemotherapy. ${ }^{19}$ In peripheral T-cell lymphoma, TP63 rearranged cases had a significantly worse overall survival. ${ }^{18}$ In lung cancer, the role of TP63 amplification and p63 expression on outcome is not clear. Massion et a $1^{14}$ suggested that TP63 amplification conferred a survival advantage to patients with non-small-cell carcinoma when compared with the overall population as well as patients with squamous cell carcinoma. Similarly, they found a strong association between the intensity of p63 expression and the odds of surviving, even after adjusting for various prognostic parameters such as stage. Furthermore, $\mathrm{Au}$ et $a l^{24}$ showed that squamous cell carcinomas expressing p63 had a better outcome when compared with p63-negative squamous cell carcinomas; however, this prognostic significance was lost when non-small-cell carcinomas were analyzed as a group. In other studies, p63 and p40 expressions did not appear to affect overall survival or disease-free survival in non-small-cell carcinoma. ${ }^{13,25}$

Given that p40 is negative in lung adenocarcinomas, the expression of p63 has been attributed to the presence of the transactivated isoform. ${ }^{15}$ However, this is not necessarily the case. The TP63 rearrangements we previously described in peripheral T-cell lymphomas encode fusion proteins homologous to $\Delta \mathrm{Np} 63$, in which they lack the transactivation domain of p63. ${ }^{18}$ In addition to lacking this transactivation domain, the native transcripts that encode $\Delta \mathrm{Np} 63$ isoforms also differ from TAp63 transcripts in which they are regulated by a distinct promoter region and encode a shortleader sequence (so-called ' $\mathrm{TA}$ *') $\mathrm{N}$-terminal to the DNA-binding domain of the resultant protein. The anti-p40 antibody recognizes this short-leader sequence. In contrast, p63 fusion proteins lack this leader sequence. In peripheral T-cell lymphomas, these proteins contain portions of the fusion partner fused directly to the DNA-binding domain of p63 (encoded by exons 4-8), and the resultant fusion protein is non-reactive with the anti-p40 antibody in lymphoma (ALF, unpublished). In the current lung cancer case, the DNA sequence data predict a fusion protein encoded by exons 1-3 of B3GALNT1 fused to a truncated TAp63 protein lacking the N-terminal portion of the transactivation domain (encoded by exon 1 of TP63). In contrast, the 4A4 antibody commonly used in immunohistochemistry is de-
Table 1 Details of the IHC and FISH results of the study and validation cases

Next-generation sequencing cases with break point in TP63

\begin{tabular}{|c|c|c|c|c|}
\hline \multirow{2}{*}{ Case \# } & \multirow{2}{*}{ Histology } & \multicolumn{2}{|c|}{ IHC\%-positive cells } & \multirow{2}{*}{$F I S H^{\mathrm{a}}$} \\
\hline & & P63 & P4O & \\
\hline 1 & AD mucinous & 80 & 0 & $1-2 \mathrm{G}, 1-2 \mathrm{R}, 2-4 \mathrm{~F}$ \\
\hline 2 & AD mucinous & 90 & 0 & $1-2 \mathrm{G}, 1-2 \mathrm{R}, 2-4 \mathrm{~F}$ \\
\hline \multicolumn{5}{|c|}{ Validation cases } \\
\hline 1 & MIA & $<5$ & 0 & ND \\
\hline 2 & AD lepidic & 60 & $<5$ & $3-6 \mathrm{~F}$ \\
\hline 3 & AD lepidic & 0 & 0 & ND \\
\hline 4 & AD lepidic & 0 & 0 & ND \\
\hline 5 & AD lepidic & $<5$ & 0 & ND \\
\hline 6 & AD lepidic & $<5$ & 0 & ND \\
\hline 7 & AD lepidic & $<5$ & 0 & ND \\
\hline \multicolumn{2}{|c|}{ Sum MIA/AD lepidic } & $1 / 7(14 \%)$ & $0(0 \%)$ & \\
\hline 8 & $\mathrm{AD}$ acinar & 20 & 0 & $\begin{array}{c}\text { A } 3-4 F \\
\text { B } 2 F\end{array}$ \\
\hline 9 & $\mathrm{AD}$ acinar & 60 & $<5$ & $3 \mathrm{~F}$ \\
\hline 10 & AD acinar & 40 & $<5$ & $3-6 \mathrm{~F}$ \\
\hline 11 & AD acinar & 30 & 0 & $\begin{array}{l}\text { A } 3-5 F \\
\text { B } 3-5 F\end{array}$ \\
\hline 12 & AD acinar & 20 & 0 & $\begin{array}{c}\text { A } 4-7 F \\
\text { B } 2 F\end{array}$ \\
\hline 13 & $\mathrm{AD}$ acinar & 20 & 0 & ND \\
\hline 14 & AD acinar & 0 & 0 & $2-3 \mathrm{~F}$ \\
\hline 15 & $\mathrm{AD}$ acinar & 0 & 0 & $2-3 \mathrm{~F}$ \\
\hline 16 & $\mathrm{AD}$ acinar & 0 & 0 & ND \\
\hline 17 & AD acinar & 0 & 0 & ND \\
\hline 18 & $\mathrm{AD}$ acinar & $<5$ & 0 & ND \\
\hline 19 & $\mathrm{AD}$ acinar & $<5$ & 0 & ND \\
\hline 20 & AD acinar & $<5$ & 0 & ND \\
\hline 21 & AD acinar & 0 & 0 & ND \\
\hline 22 & $\mathrm{AD}$ acinar & 0 & 0 & ND \\
\hline 23 & AD acinar & $<5$ & 0 & ND \\
\hline 24 & AD acinar & $<5$ & 0 & ND \\
\hline 25 & AD papillary & 0 & 0 & ND \\
\hline \multicolumn{2}{|c|}{ Sum AD acinar/papillary } & $6 / 18(33 \%)$ & $0(0 \%)$ & \\
\hline 26 & AD cribriform & 50 & 0 & $3-4 \mathrm{~F}$ \\
\hline 27 & AD cribriform & 20 & 0 & $3-6 \mathrm{~F}$ \\
\hline 28 & AD cribriform & 30 & 0 & $3-5 \mathrm{~F}$ \\
\hline 29 & AD cribriform & $<5$ & 0 & ND \\
\hline 30 & AD cribriform & 0 & 0 & ND \\
\hline 31 & AD cribriform & $<5$ & 0 & ND \\
\hline 32 & AD solid & 20 & $<5$ & $3-4 \mathrm{~F}$ \\
\hline 33 & AD solid & 30 & $<5$ & $\begin{array}{l}\text { A } 3 F \\
\text { B } 2 F\end{array}$ \\
\hline 34 & AD solid & 30 & 0 & $3-6 \mathrm{~F}$ \\
\hline 35 & AD solid & 0 & 0 & ND \\
\hline 36 & AD solid & 0 & 0 & ND \\
\hline 37 & AD solid & $<5$ & 0 & ND \\
\hline 38 & AD solid & $<5$ & 0 & ND \\
\hline 39 & AD solid & 0 & 0 & ND \\
\hline 40 & AD solid & 0 & 0 & ND \\
\hline 41 & AD solid & $<5$ & 0 & ND \\
\hline 42 & AD solid & 0 & 0 & ND \\
\hline \multicolumn{2}{|c|}{ Sum AD cribriform/solid } & $6 / 17(35 \%)$ & $0(0 \%)$ & \\
\hline 43 & AD mucinous & 0 & 0 & ND \\
\hline 44 & AD mucinous & $<5$ & 0 & ND \\
\hline \multicolumn{2}{|c|}{ Sum AD mucinous } & $0 / 2(0 \%)$ & $0(0 \%)$ & \\
\hline
\end{tabular}

a Abbreviations: FISH, Fluorescence in-situ hybridization; IH, immunohistochemistry; ND, not done.FISH signal pattern is indicated as follows: F, fusion signal (intact TP63); G, green signal (3' TP63); R, red signal ( $5^{\prime}$ TP63).

rived against an epitope spanning regions of the p63 protein common to all native isoforms as well as the described fusion proteins. Thus, this antibody 

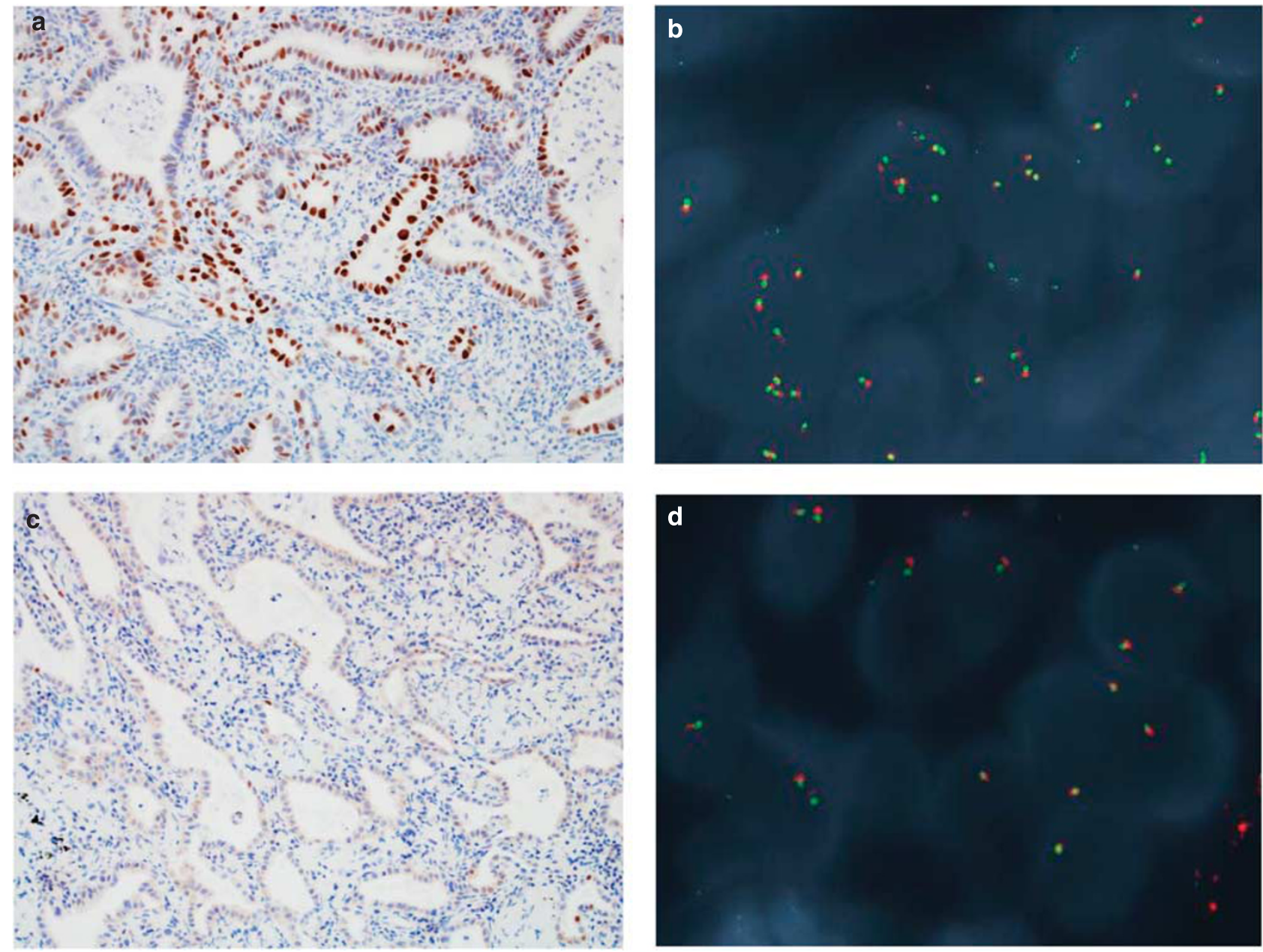

Figure 3 (a) Adenocarcinoma with focal expression of p63 (p63, × 200). (b) FISH demonstrates extra copies of the intact TP63 locus in this area. (c and d) In contrast, in the area of adenocarcinoma with no expression of p63, only two copies of TP63 are identified by FISH.

cannot distinguish native $\Delta \mathrm{Np63}$ from TAp63 isoforms, nor native protein from fusion protein. Therefore, TP63 rearrangements possibly could explain expression of p63 in a subset of adenocarcinoma, particularly cases with diffuse expression as seen in our case.

p63 expression in lung adenocarcinoma could also be explained by amplification of TP63. In our study, all adenocarcinomas expressing p63 that did not have rearrangements of TP63 demonstrated increased copy numbers of TP63. Interestingly, this correlated with the focal expression of p63. Indeed, in cases where FISH could be scored separately in the p63-positive and p63-negative areas, increased copy numbers were observed only in the p63-positive areas. Additionally, no increased copy numbers was identified in the two p63/ p40-negative adenocarcinomas. The correlation between p63 expression and TP63 amplification is well demonstrated in lung squamous cell carcinoma and has also been reported in lung adenocarcinoma, although with lower frequency. ${ }^{5,14}$ In our study, a third of the adenocarcinoma demonstrated p63 expression and increased copy numbers of TP63. This is in contrast to the $0-11 \%$ reported frequency of TP63 amplification. ${ }^{5,14}$ Reported expression of p63 in adenocarcinoma has been more variable but similar to our results, ranging up to $31 \%,{ }^{10-17}$ with most recent studies focusing only on expression and not amplification, which could explain the lower reported amplification frequency.

\section{Acknowledgments}

This work was supported by the Mayo Clinic Center for Individualized Medicine (CIM) Biomarker Discovery (BMD) Program. ALF is a Damon Runyon Clinical Investigator supported by the Damon Runyon Cancer Research Foundation (CI-48-09).

\section{Disclosure/conflict of interest}

The authors declare no conflict of interest. 


\section{References}

1 Senoo M, Seki N, Ohira M, et al. A second p53-related protein, p73l, with high homology to p73. Biochem Biophys Res Commun 1998;248:603-607.

2 Brunner HG, Hamel BCJ, van Bokhoven H. The p63 gene in EEC and other syndromes. J Med Genet 2001;39:377-381.

3 Osada M, Ohba M, Kawahara C, et al. Cloning and functional analysis of human p51, which structurally and functionally resembles p53. Nat Med 1998;4:839843.

4 Kato S, Shimada A, Osada M, et al. Effects of $p 51 / p 63$ missense mutations on transcriptional activities of $p 53$ downstream gene promoters. Cancer Res 1999;59: 5908-5911.

5 Hibi K, Trink B, Patturajan M, et al. AIS is an oncogene amplified in squamous cell carcinoma. Proc Natl Acad Sci USA 2000;97:5462-5467.

$6 \mathrm{Su} \mathrm{X,} \mathrm{Chakravarti} \mathrm{D,} \mathrm{Flores} \mathrm{ER.} \mathrm{p63} \mathrm{steps} \mathrm{into} \mathrm{the}$ limelight: crucial roles in the suppression of tumorigenesis and metastasis. Nat Med 2013;13:136-142.

7 Hagiwara K, McMenamin G, Miura K, et al. Mutational analysis of the $p 63 / p 73 L p 51 / p 40 / C U S P / K E T$ gene in human cancer cell lines using intronic primers. Cancer Res 1999;59:4165-4169.

8 Sunahara M, Shishikura T, Takahashi M, et al. Mutational analysis of $p 51 A / T a p 63 \gamma$, a p53 homolog, in non-small cell lung cancer and breast cancer. Oncogene 1999;18:3761-3765.

9 Nylander K, Vojtesek B, Nenutil R, et al. Differential expression of p63 isoforms in normal tissues and neoplastic cells. J Pathol 2002;198:417-427.

10 Kaufmann O, Fietze E, Mengs J, et al. Value of p63 and CK5/6 as immunohistochemical markers for the differential diagnosis of poorly differentiated and undifferentiated carcinomas. Am J Clin Pathol 2001;116: 823-830.

11 Kargi A, Gurel D, Tuna B. The diagnostic value of TTF1 , CK5/6, and p63 immunostaining in the classification of lung carcinoma. Appl Immunohistochem Mol Morphol 2007;15:415-420.

12 Mukhopadhyay S, Katzenstein A-L. Subclassification of non-small cell lung carcinomas lacking morphologic differentiation on biopsy specimens: utility of an immunohistochemical panel containing TTF-1, Napsin A, p63, and CK5/6. Am J Surg Pathol 2011;35:15-25.

13 Pelosi G, Pasini F, Olsen Stenholm C, et al. p63 immunoreactivity in lung cancer: yet another player in the development of squamous cell carcinomas? J Pathol 2002;198:100-109.

14 Massion PP, Taflan PM, Jamshedur Rahman SM, et al. Significance of p63 amplification and overexpression in lung cancer development and prognosis. Cancer Res 2003;63:7113-7121.

15 Nonaka D. A study of $\Delta \mathrm{Np} 63$ expression in lung nonsmall cell carcinomas. Am J Surg Pathol 2012;36: 895-899.

16 Rekhtman N, Ang DC, Sima CS, et al. Immunohistochemical algorithm for differentiation of lung adenocarcinoma and squamous cell carcinoma based on large series of whole-tissue sections with validation in small specimens. Mod Pathol 2011;24: 1348-1359.

17 Bishop JA, Teruya-Feldstein J, Westra WH, et al. p40 $(\Delta \mathrm{Np} 63)$ is superior to p63 for the diagnosis of pulmonary squamous cell carcinoma. Mod Pathol 2012;25:405-415.

18 Vasmatzis G, Johnson SH, Knudson RA, et al. Genomewide analysis reveals recurrent structural abnormalities of TP63 and other p53-related genes in peripheral T-cell lymphomas. Blood 2012;120:2280-2289.

19 Scott DW, Mungall KL, Ben-Neriah S, et al. TBL1XR1/ TP63: a novel recurrent gene fusion in B-cell nonHodgkin lymphoma. Blood 2012;119:4949-4952.

20 Travis WD, Brambilla E, Muller-Hermelink KH, Harris CCeds.Tumors of the Lung, Pleura, Thymus and Heart. WHO classification of tumors. IARC Press: Lyon, 2004;37-46.

21 Travis WD, Brambilla E, Noguchi M, et al. International Association for the Study of Lung Cancer/ American Thoracic Society/European Respiratory Society International Multidisciplinary Classification of Lung Adenocarcinoma. J Thorac Oncol 2011;6: 244-285.

22 Murphy SJ, Cheville JC, Zarei S, et al. Mate pair sequencing of whole-genome-amplified DNA following laser capture microdissection of prostate cancer. DNA Res 2012;19:395-406.

23 Feldman AL, Dogan A, Smith DI, et al. Discovery of recurrent $\mathrm{t}(6,7)(\mathrm{p} 25$ 3,q32 3) translocations in ALK negative anaplastic large cell lymphomas by massively parallel genomic sequencing. Blood 2011;117: 915-919.

24 Au NHC, Cheang M, Hunstman DG, et al. Evaluation of immunohistochemical markers in non-small cell lung cancer by unsupervised hierarchical clustering analysis: a tissue microarray study of 284 cases and 18 markers. J Pathol 2004;204:101-109.

25 Iwata T, Uramoto H, Sugio K, et al. A lack of prognostic significance regarding $\Delta \mathrm{Np} 63$ immunoreactivity in lung cancer. Lung Cancer 2005;50:67-73. 